\title{
In Utero and Childhood Polybrominated Diphenyl Ether (PBDE) Exposures and Neurodevelopment in the CHAMACOS Study
}

\author{
Brenda Eskenazi, ${ }_{1}^{1}$ Jonathan Chevrier, ${ }^{1}$ Stephen A. Rauch, ${ }^{1}$ Katherine Kogut, ${ }^{1}$ Kim G. Harley, ${ }^{1}$ Caroline Johnson, ${ }^{1}$ \\ Celina Trujillo, ${ }^{1}$ Andreas Sjödin, ${ }^{2}$ and Asa Bradman ${ }^{1}$
}

${ }^{1}$ Center for Environmental Research and Children's Health, School of Public Health, University of California at Berkeley, Berkeley, California, USA; ${ }^{2}$ Division of Laboratory Sciences, National Center for Environmental Health, Centers for Disease Control and Prevention, Atlanta, Georgia, USA

BACKGROUND: California children's exposures to polybrominated diphenyl ether flame retardants (PBDEs) are among the highest worldwide. PBDEs are known endocrine disruptors and neurotoxicants in animals.

ОвJестіVE: Here we investigate the relation of in utero and child PBDE exposure to neurobehavioral development among participants in CHAMACOS (Center for the Health Assessment of Mothers and Children of Salinas), a California birth cohort.

Methods: We measured PBDEs in maternal prenatal and child serum samples and examined the association of PBDE concentrations with children's attention, motor functioning, and cognition at $5(n=310)$ and 7 years of age $(n=323)$.

RESULTS: Maternal prenatal PBDE concentrations were associated with impaired attention as measured by a continuous performance task at 5 years and maternal report at 5 and 7 years of age, with poorer fine motor coordination-particularly in the nondominant—at both age points, and with decrements in Verbal and Full-Scale IQ at 7 years. PBDE concentrations in children 7 years of age were significantly or marginally associated with concurrent teacher reports of attention problems and decrements in Processing Speed, Perceptual Reasoning, Verbal Comprehension, and Full-Scale IQ. These associations were not altered by adjustment for birth weight, gestational age, or maternal thyroid hormone levels.

ConCLUSIONS: Both prenatal and childhood PBDE exposures were associated with poorer attention, fine motor coordination, and cognition in the CHAMACOS cohort of school-age children. This study, the largest to date, contributes to growing evidence suggesting that PBDEs have adverse impacts on child neurobehavioral development.

KEY WORDS: ADHD, attention, biomarkers, children, cognitive development, flame retardants, human exposure, intelligence quotient, Mexican, motor, neurodevelopment, prenatal. Environ Health Perspect 121:257-262 (2013). http://dx.doi.org/10.1289/ehp.1205597 [Online 15 November 2012]

Polybrominated diphenyl ether (PBDEs) flame retardant chemicals, used in the manufacture of furniture, infant products, and electronics, are ubiquitous in U.S. households (Sjödin et al. 2008). An unintended consequence of California's Technical Bulletin 117 (TB 117) —a fire safety law promulgated in the 1970s which requires that furniture, baby, and other household products resist open flame (California Department of Consumer Affairs 2000; Zota et al. 2008) - is that PBDE concentrations in California children are now among the highest measured worldwide (Eskenazi et al. 2011). Until 2005, the predominant chemical flame retardant used to comply with TB 117 was pentaBDE (comprising congeners BDEs 47, 99, 100, and 153). Although pentaBDE was banned in California and phased out by the manufacturer in 2004, pentaBDEs continue to leach from older household items. Exposure is also perpetuated by decaBDEs, still used in many electronic products, which can break down into lower-brominated congeners (Noyes et al. 2011). Because PBDEs are semivolatile and not chemically bound to substrates, they migrate into house dust, placing young children, who crawl on the floor and exhibit frequent hand-to-mouth behaviors, at risk of higher exposures (Stapleton et al. 2008).

PBDEs are endocrine-disrupting compounds with half-lives in humans ranging from 2 to 12 years (Geyer et al. 2004). Recent research suggests that PBDE exposures are associated with altered thyroid hormone levels in pregnant women (Chevrier et al. 2010) and infants (Herbstman et al. 2008), and negatively associated with birth weight (Harley et al. 2011). Research also suggests possible neurotoxic effects of in utero and early childhood exposure to PBDEs (Chao et al. 2007; Gascon et al. 2011, 2012; Herbstman et al. 2010; Hoffman et al. 2012; Roze et al. 2009). Herbstman et al. (2010) reported significant decrements in motor and mental development at ages 1-6 years associated with in utero PBDE exposures in New York children $(n=100)$. In a study of 625 - to 6-year-old Dutch children, Roze et al. (2009) reported that in utero exposure levels were negatively associated with fine motor coordination and sustained attention, although improved coordination and visual perception and fewer internalizing and externalizing behaviors.
Recently, Gascon et al. (2011) reported that 4-year-old Spanish children with detectable blood concentrations of BDE-47 were significantly more likely to demonstrate attention symptoms [DSM-IV (Diagnostic and Statistical Manual of Mental Disorders, 4th ed.) (American Psychiatric Association 1994) scores $>80$ th percentile] than less-exposed peers, but not motor or cognitive deficits. Cord blood BDE-47 concentrations were not associated with any neurobehavioral parameters at 4 years of age. Hoffman et al. (2012) found a positive association between breast milk levels of BDEs 47, 99, and 100 and externalizing behaviors, specifically activity/ impulsivity behaviors in 22030 -month-olds.

In this analysis, we examined the relationship of prenatal maternal and child PBDE concentrations with attention, cognition, and motor development in California children at 5 and 7 years of age.

\section{Methods}

The Center for the Health Assessment of Mothers and Children of Salinas (CHAMACOS) is a longitudinal birth cohort study of predominantly Mexican-American families in California's Salinas Valley. Detailed methods for CHAMACOS are published

Address correspondence to B. Eskenazi, Center for Environmental Research and Children's Health (CERCH), School of Public Health, University of California at Berkeley, 1995 University Ave., Suite 265, Berkeley, CA 94720 USA. Telephone: (510) 642-3496. E-mail: eskenazi@berkeley.edu

Supplemental Material is available online (http:// dx.doi.org/10.1289/ehp.1205597).

We acknowledge the CHAMACOS staff, students, community partners, and participants, as well as N. Holland and staff for assistance in specimen management.

This publication was made possible by research supported by grants RD 83171001 and RD 826709 from the U.S. Environmental Protection Agency (EPA), and PO1 ES009605 and RO1 ES015572 from the National Institute of Environmental Health Sciences (NIEHS). Additional funding was provided by the University of California Institute for Mexico and the United States (UC MEXUS).

The contents of this publication are solely the authors' responsibility and do not necessarily represent the official views of the UC MEXUS, NIEHS, National Institutes of Health, the U.S. EPA, or the Centers for Disease Control and Prevention.

The authors declare they have no actual or potential competing financial interests.

Received 9 June 2012; accepted 7 November 2012. 
elsewhere (Eskenazi et al. 2004, 2006). Eligible pregnant women ( $\geq 18$ years old, $<20$ weeks gestation, Spanish- or English-speaking, qualifying for low-income health insurance, and planning to deliver at the public hospital) were recruited between October 1999 and October 2000 from community clinics. The cohort included 601 women, 526 of whom delivered live-born singletons.

Women were interviewed twice during pregnancy (at -13 and 26 weeks gestation), after delivery, and when children were 6 months old, and 1, 2, 3.5, 5, and 7 years old. Mothers completed the Peabody Picture Vocabulary Test (PPVT) or Test de Vocabulario en Imágenes Peabody (TVIP) of verbal intelligence (Dunn and Dunn 1981) at the 6-month visit and the Center for Epidemiologic Studies Depression Scale (CES-D) (Radloff 1977) at the 1-year visit. Age-appropriate versions of the HOME (Home Observation for Measurement of the Environment) survey were completed at most postdelivery visits (Caldwell and Bradley 1984). Birth weight and gestational duration were abstracted from medical records.

Neurobehavioral assessments were performed by bilingual psychometricians, and children were assessed in their dominant language. A total of 310 children were assessed at 5 years (mean $=60.0 \pm 2.6$ months) and 323 at 7 years $(85.2 \pm 2.9$ months $)$. The present analysis excludes four children with autism, Down syndrome, cerebral palsy/ hydrocephalus, or deafness and 63 children who lacked PBDE measurements.

Compared with children in the cohort who were not followed, children included in the present analyses were more likely to be female and born full term, with mothers who were older, breastfed longer, and were less likely to smoke or drink during pregnancy (data not shown). They did not differ according to other sociodemographic characteristics or by their maternal prenatal PBDE levels [median $=24.9 \mathrm{ng} / \mathrm{g}$ lipid; interquartile range (IQR) 14.0-42.1] for those followed versus $23.8 \mathrm{ng} / \mathrm{g}$ lipid (IQR = 14.9-41.3) for those not followed].

Mothers provided written informed consent at both visits, and children provided verbal assent at 7 years of age. Study activities were approved by the University of California at Berkeley (UC) Committee for the Protection of Human Subjects. A technical assistance agreement was established between the Division of Laboratory Sciences at the National Center for Environmental Health, Centers for Disease Control and Prevention (CDC), and UC Berkeley.

Attention. At the 5-year visit, mothers completed the Child Behavior Checklist (CBCL)/1.5-5 (CBCL) (Achenbach and Rescorla 2000). We analyzed two subscales as continuous raw scores: the Attention Problems scale and the DSM-IV-oriented Attention Deficit/Hyperactivity Disorder (ADHD) Problems scale. We also analyzed a "borderline clinical range" ( $\geq 93$ rd percentile in the standardization sample) indicator variable for each scale (Achenbach and Rescorla 2000). In addition, children were assessed on the Conners' Kiddie Continuous Performance Test (K-CPT) (Conners and Staff 2001), a 7 -min computerized vigilance task that assesses reaction time, accuracy, and impulse control. We determined continuous $T$-scores (standardized to a nonclinical population) for errors of commission, errors of omission, and hit reaction time (Conners and Staff 2001). We also examined the continuous ADHD Confidence Index score, which indicates the probability that children are correctly classified as having clinical ADHD, and a binary variable indicating a Confidence Index score $\geq 70$ th percentile.

At child's age 7 years, mothers and teachers completed the Conners' ADHD/ DSM-IV Scales (CADS) (Conners 2001) and the Behavior Assessment System for Children, 2nd edition (BASC) (Reynolds and Kamphaus 2004). CADS data from four subscales (Conners ADHD index score, and DSM-IV-based Inattentive, Hyperactive/ Impulsive, and Total ADHD scores) were analyzed both as continuous, standardized scores ( $T$-scores; mean $\pm \mathrm{SD}=50 \pm 10)$ and as a binary variable indicating scores in the "Moderately" or "Markedly Atypical" range (T-score $\geq 65$ ) (Conners 2001). BASC data from Hyperactivity and Attention Problems subscales were analyzed as standardized $T$-scores and as a binary "at-risk" or "clinically significant" variable ( $T$-score $\geq 60)$ (Reynolds and Kamphaus 2004).

Motor function. At ages 5 and 7 years, children's gross motor skills were assessed using select subscales of the McCarthy Scales of Children's Abilities (McCarthy 1972). Their fine motor dexterity was assessed with a pegboard test (Wide Range Assessment of Visual Motor Ability; WRAVMA) (Adams and Sheslow 1995) (age-standardized mean $=100 \pm 15)$ and with a finger-tapping task [at 5 years: Behavioral Assessment and Research System (BARS) (Rohlman et al. 2003); and at 7 years: Reitan Neuropsychology Laboratory (Tucson, AZ)]. We standardized McCarthy gross motor and finger tap scores within our study population $(z$-scores, mean $=0 \pm 1$ ).

Cognitive functioning. At 5 years of age, children completed tests of receptive verbal intelligence in both English and Spanish using the PPVT and TVIP, respectively (Dunn and Dunn 1981). We analyzed children's continuous standardized scores $($ mean $=100 \pm 15)$ in their language of best performance. We assessed children's performance intelligence (PIQ) with the Wechsler Preschool and Primary Scale of Intelligence, 3rd edition (WPPSI-III) $($ mean $=100 \pm 15)$.

At age 7 years, children were assessed on four subdomains of the Wechsler Intelligence Scale for Children-Fourth Edition (WISC-IV) (Wechsler 2003): Verbal Comprehension, Perceptual Reasoning, Working Memory, and Processing Speed. A Full-Scale IQ was also calculated (mean $=100 \pm 15$ for the FullScale IQ and all components).

Other questions. Mothers were also asked "Has a doctor, nurse, psychologist or teacher ever told you that your child might have 1) attention problems? or 2) learning problems?" Teachers were asked "Do you have any specific concerns about this student (in terms of) 1) emotional problems, 2) behavioral problems, or 3) learning problems?"

PBDE exposure assessment. Blood samples were collected by venipuncture from mothers during pregnancy $($ mean $=26.7 \pm 2.6$ weeks gestation, $n=219)$ or at delivery $(n=60)$, and from children at the 7 -year visit $(n=272)$. PBDE serum levels in women with data at both time points were very strongly correlated (Pearson $r \geq 0.98, p<0.001$ )]. Samples were immediately processed and stored at $-80^{\circ} \mathrm{C}$ until shipment on dry ice to the CDC (Atlanta, GA). Samples were analyzed at CDC for 10 congeners (BDEs 17, 28, 47, 66, 85, $99,100,153,154$, and 183) using gas chromatography isotope dilution high-resolution mass spectrometry (Sjödin et al. 2004). PBDE concentrations are expressed on a serum lipid basis (nanograms per gram lipids). Total serum lipid concentrations were determined based on the measurement of triglycerides and total cholesterol using standard enzymatic methods (Roche Chemicals, Indianapolis, IN) (Phillips et al. 1989). The limits of detection (LODs) for BDE-47 ranged from 0.3 to $2.6 \mathrm{ng} / \mathrm{g}$ lipids for maternal samples, and 0.4 to $0.8 \mathrm{ng} / \mathrm{g}$ lipids for child samples. For all other congeners, LODs ranged between 0.2 and $0.7 \mathrm{ng} / \mathrm{g}$ lipids for maternal and 0.3 and $5.6 \mathrm{ng} / \mathrm{g}$ lipids for child samples, respectively. Quality control samples (blanks and spikes) were included in each run.

We used the sum of BDEs 47, 99, 100, and 153 congeners as our primary exposure measure. Values < LOD were assigned the machine-read value if a signal was detected. If not, all concentration levels < LOD were imputed at random based on a log-normal probability distribution using maximum likelihood estimation (Lubin et al. 2004).

We assessed maternal exposure to organophosphate (OP) insecticides as measured by dialkyl phosphate (DAP) metabolites in maternal urine (at 13 and 26 weeks gestation), using an isotope dilution gas chromatographytandem mass spectrometry method (Bradman 
et al. 2005; Bravo et al. 2002); lead in maternal prenatal and cord blood samples, using graphite furnace atomic absorption spectrophotometry; polychlorinated biphenyls (PCBs) in maternal serum using high-resolution gas chromatography/high-resolution mass spectrometry with isotope dilution quantification (Barr et al. 2003); and maternal thyroid stimulating hormone (TSH; using immunochemiluminometric assay) and free thyroxine $\left(\mathrm{T}_{4}\right.$; using direct equilibrium dialysis followed by radioimmunoassay) (Bayer ADVIA Centaur system; Siemens Healthcare Diagnostics, Deerfield, IL) at 26 weeks gestation (Chevrier et al. 2010; Nelson and Tomei 1988).

Data analysis. PBDE levels were expressed on the $\log _{10}$ scale. To determine the shape of the dose-response function, we ran generalized additive models using cubic splines. If nonlinearity was detected $(p<0.10)$, additional models were run with categorized PBDE concentrations (quartiles). We re-ran all final models with PBDE concentrations expressed on a serum basis (picograms per gram serum) with total serum lipids as a covariate. We also ran models with the sum of all 10 PBDE congeners; individually for each of the four primary congeners $(47,99,100$, and 153); and excluding outliers (defined as being $\geq 3.5 \mathrm{SD}$ away from the mean for $\log _{10}$ PBDEs or the outcome).

Variables were identified as potential confounders based on their relationship to neurodevelopment. We examined the following [see Supplemental Material, Table S3 for categories (http://dx.doi.org/10.1289/ ehp.1205597)]: maternal age, education, years in the United States, marital status, work outside the home, use of alcohol and tobacco during pregnancy, depression (CES-D), parity, and PPVT or TVIP score; housing density, household poverty, pregnancy exposure to environmental tobacco smoke, number of children in the home, father's presence in the home, and HOME score at 6 months and 7 years; preschool and out-of-home child care attendance; psychometrician, location, and language of assessment; and child sex, birth weight, preterm delivery status, and handedness (motor outcomes only). Missing values $(<10 \%)$ for covariates were imputed by randomly selecting a value from the dataset.

We built separate models for attention, cognition, and motor outcomes, and used the same model for all outcomes within a category. In addition to child's sex and months of age (continuous), final models included all covariates that changed the coefficient for the main exposure and any outcome within the group by $>10 \%$. The covariates maintained in the models are listed in the footnote of the respective tables.

For sensitivity analyses, we adjusted for birth weight, gestational age at birth, maternal thyroid hormone (TSH and free $\mathrm{T}_{4}$ ), DAPs, lead, and PCBs in separate models (Chevrier et al. 2010; Harley et al. 2011). We evaluated effect modification by child sex. In addition, we included maternal and child PBDE levels in the same models, although doing so reduced the sample size $(n=214)$.

Main effects were considered statistically significant with $p<0.05$ based on two-tailed tests, and interactions were considered significant if $p<0.10$. All analyses were conducted with STATA version 10.1 (StataCorp, College Station, TX).

\section{Results}

For both mothers and children, BDE congeners 47, 99, 100, and 153 had detection frequencies $>97 \%$ and dominated the total measure of concentration, with BDE-47 in the highest concentration [for maternal and child measures, see Supplemental Material, Tables S1 and S2, respectively (http://dx.doi. org/10.1289/ehp.1205597)]. Children's PBDE levels were more than three times higher than the mothers' for the sum of four congeners, and detection frequencies for most other congeners were also substantially higher in children (Bradman et al. 2012; Castorina et al. 2011; Eskenazi et al. 2011). The correlation between maternal and child $\Sigma$ PBDE levels was $0.27(p<0.001)$; the correlation for individual congeners ranged from 0.21 for BDE-99 to 0.30 for BDE-153. Supplemental Material, Table S3, presents the distribution of demographic characteristics for children in the study sample and the geometric means (GM) of maternal and child $\Sigma$ PBDE concentrations by covariates. Supplemental Material, Table S4, summarizes neurobehavioral scores for the study population.

Correlations between reports by teachers and parents concerning attention at 7 years of age, and between measures of attention, cognition, and motor skills, were moderate. For example, correlations between maternal and teacher report on the CADS ranged from $r=0.2-0.3(p<0.01)$. Similar measures of attention on the BASC and CADS within a reporter (mother/teacher) were more strongly correlated-for example, $r=0.5$ to $0.8, p<0.001$ for maternal report and $r=0.7$ to $0.8, p<0.001$ for teacher report. Maternal and teacher CADS scores were negatively correlated with WISC Full-Scale IQ scores $(r=-0.2$ to $-0.3, p<0.001)$. Motor skills outcomes tended to be positively correlated with IQ scores $(r=0.1$ to 0.4 , several $p<0.001)$ and negatively correlated with attention outcomes $(r=-0.05$ to -0.2 , several $p<0.01)$ (data not shown).

Attention. At child age 5 years, maternal prenatal $\Sigma$ PBDE concentrations (for the 4 main congeners) were marginally associated $(p<0.10)$ with maternally reported CBCL scores above the 93rd percentile for attention problems [adjusted odds ratio (aOR) for a 10 -fold increase in $\Sigma \mathrm{PBDE}=4.6 ; 95 \% \mathrm{CI}$ : 0.9, 24.5] [see Supplemental Material, Table S5 (http://dx.doi.org/10.1289/ ehp.1205597)], and strongly associated with both errors of omission scores and ADHD Confidence Index scores on the K-CPT (Table 1). Quartile categorization suggested that both errors of omission and the ADHD Confidence Index were primarily elevated in children with mothers in the highest quartile of $\Sigma$ PBDE exposure (> $42 \mathrm{ng} / \mathrm{g}$ ) (Figure 1).

At child age 7 years, maternal $\Sigma$ PBDE exposure was associated with maternally reported $\mathrm{ADHD}$ Index scores on the CADS $(\beta=2.9 ; 95 \%$ CI: $0.7,5.2)$, DSM-IV Total scores $(\beta=2.6 ; 95 \% \mathrm{CI}: 0.2,5.0)$, and DSM-IV Inattention scale scores $(\beta=2.2$; 95\% CI: 0.0, 4.5) (Table 1). Although there was evidence of nonlinearity for the DSM measures, quartile categorization showed no clear trends (Figure 1). Maternal exposure was also related to somewhat higher odds of a mother having been told that her child had attention problems $(\mathrm{aOR}=2.3$; 95\% CI: 0.9 , 5.8 ), and to teacher reports of child behavior problems $(\mathrm{aOR}=2.5$; 95\% CI: $1.1,6.0)$ [see Supplemental Material, Table S5 (http:// dx.doi.org/10.1289/ehp.1205597)]. However, there were no associations between maternal $\triangle \mathrm{PBDE}$ and teacher ratings on the CADS or BASC, or maternal ratings on the BASC, for continuous or dichotomous outcomes.

By contrast, child PBDE concentrations were associated with reports of attention problems from teachers, but not from mothers. Specifically, child $\Sigma$ PBDEs were associated with more adverse teacher reports on CADS ADHD Index, CADS DSM-IV Total, CADS DSM-IV Inattentive, BASC Hyperactivity, and BASC Attention Problems scales [Table 1; see also Supplemental Material, Table S5 (http://dx.doi.org/10.1289/ehp.1205597)]. Associations were particularly pronounced for some of the dichotomous outcomes: Every 10 -fold increase in child $\Sigma$ PBDE level was associated with 4.5 and 5.5 times higher odds of the child being rated by the teacher as being in the "moderately or markedly atypical" range on CADS DSM-IV Hyperactive/Impulsive subscale $(95 \%$ CI: $1.2,16.6)$ and DSM-IV Total subscale (95\% CI: 1.5, 20.3), respectively (see Supplemental Material, Table S5).

Motor function. We observed little evidence of association between either maternal or child $\Sigma$ PBDE serum concentrations and gross motor performance on McCarthy scales (Table 2). However, maternal $\Sigma$ PBDEs were related to poorer performance on the WRAVMA pegboard at both 5 and 7 years, particularly for the nondominant hand. For the 5-year-olds, this relationship was observed primarily for the nondominant hand among boys (boys: $\beta=-12.1$; 95\% CI: $-19.4,-4.7$; girls: $\beta=0.8$; 
95\% CI: $\left.-6.8,8.5 ; p_{\text {interaction }}=0.09\right)$, whereas at age 7 , it was seen mainly in the dominant hand in girls (boys: $\beta=-2.7 ; 95 \% \mathrm{CI}$ : -10.8 , 5.4; girls $\beta=-8.1$; 95\% CI: $-16.3,0.1$; $\left.p_{\text {interaction }}=0.08\right)$. Associations between maternal $\Sigma$ PBDEs and pegboard performance at
7 years showed evidence of nonlinearity, with nonsignificantly poorer performance in children of mothers in the highest quartile of exposure (Figure 1). At 5 but not 7 years of age, maternal $\Sigma$ PBDEs were also inversely associated with dominant-hand finger taps (Table 2).

Table 1. Adjusted linear models for attention-related outcome scores in CHAMACOS children at 5 and 7 years of age, per 10 -fold increase in maternal prenatal and child $\sum$ PBDE concentration (ng/g, lipidadjusted).

\begin{tabular}{|c|c|c|c|c|}
\hline \multirow[b]{2}{*}{ Outcome } & \multicolumn{2}{|c|}{ Maternal $\sum \mathrm{PBDE}^{\mathrm{a}, \mathrm{c}}$} & \multicolumn{2}{|c|}{ Child $\sum$ PBDE ${ }^{b, c}$} \\
\hline & $n$ & $\beta(95 \% \mathrm{Cl})$ & $n$ & $\beta(95 \% \mathrm{Cl})$ \\
\hline \multicolumn{5}{|l|}{ Assessment of 5-year-olds } \\
\hline \multicolumn{5}{|l|}{ CBCL (raw score) } \\
\hline Attention problems & 249 & $0.1(-0.4,0.6)$ & & \\
\hline ADHD & 249 & $0.4(-0.5,1.2)$ & & \\
\hline \multicolumn{5}{|l|}{$\mathrm{K}$-CPT (T-score) } \\
\hline Errors of omission & 246 & $5.8(1.5,10.1)^{* *, \#}$ & & \\
\hline Errors of commission & 246 & $-0.5(-3.7,2.7)$ & & \\
\hline ADHD Confidence Index & 233 & $7.0(1.6,12.4)^{* *, \#}$ & & \\
\hline \multicolumn{5}{|l|}{ Assessment of 7-year-olds } \\
\hline \multicolumn{5}{|c|}{ Conner's rating scale (CADS)-maternal report ( $T$-score) } \\
\hline ADHD index & 266 & $2.9(0.7,5.2)^{* *}$ & 270 & $1.0(-1.9,3.9)$ \\
\hline DSM-IV total scale & 266 & $2.6(0.2,5.0)^{* *, \#}$ & 270 & $1.4(-1.5,4.4)$ \\
\hline Inattentive subscale & 266 & $2.2(0.0,4.5)^{* *, \#}$ & 270 & $0.7(-2.1,3.5)^{\#}$ \\
\hline Hyperactive/Impulsive subscale & 266 & $1.6(-0.8,4.1)$ & 270 & $1.9(-1.1,5.0)$ \\
\hline \multicolumn{5}{|l|}{ BASC-2-maternal report (T-score) } \\
\hline Hyperactivity scale & 257 & $1.0(-1.5,3.6)$ & 269 & $0.5(-2.6,3.5)$ \\
\hline Attention Problems scale & 257 & $0.5(-1.2,2.1)$ & 269 & $-0.1(-2.1,1.9)$ \\
\hline \multicolumn{5}{|c|}{ Conner's rating scale (CADS)-teacher report ( $T$-score) } \\
\hline ADHD index & 213 & $2.4(-1.4,6.1)$ & 219 & $4.6(-0.4,9.6)^{*}$ \\
\hline DSM-IV total scale & 212 & $1.8(-1.4,5.0)$ & 217 & $4.0(-0.3,8.3)^{*}$ \\
\hline Inattentive subscale & 216 & $1.2(-1.6,3.9)$ & 221 & $3.7(0.1,7.4)^{* *}$ \\
\hline Hyperactive/Impulsive subscale & 216 & $1.7(-1.7,5.0)$ & 221 & $3.5(-1.1,8.0)$ \\
\hline \multicolumn{5}{|l|}{ BASC-2-teacher report (T-score) } \\
\hline Hyperactivity scale & 217 & $1.8(-1.3,4.9)$ & 222 & $4.8(0.5,9.0)^{* *}$ \\
\hline Attention Problems scale & 257 & $0.7(-1.3,2.7)$ & 222 & $2.9(0.4,5.5)^{* *}$ \\
\hline
\end{tabular}

a Maternal PBDE models control for child's age at assessment, sex, maternal education, number of children in the home, and psychometrician (5-year assessments only). ${ }^{b}$ Child PBDE models control for child's age at assessment, sex, and parity. ${ }^{c}$ Sum of four PBDE congeners: BDEs $47,99,100$, and 153. ${ }^{*} p<0.1$. ${ }^{* *} p \leq 0.05$. "Digression from linearity at $p<0.10$.

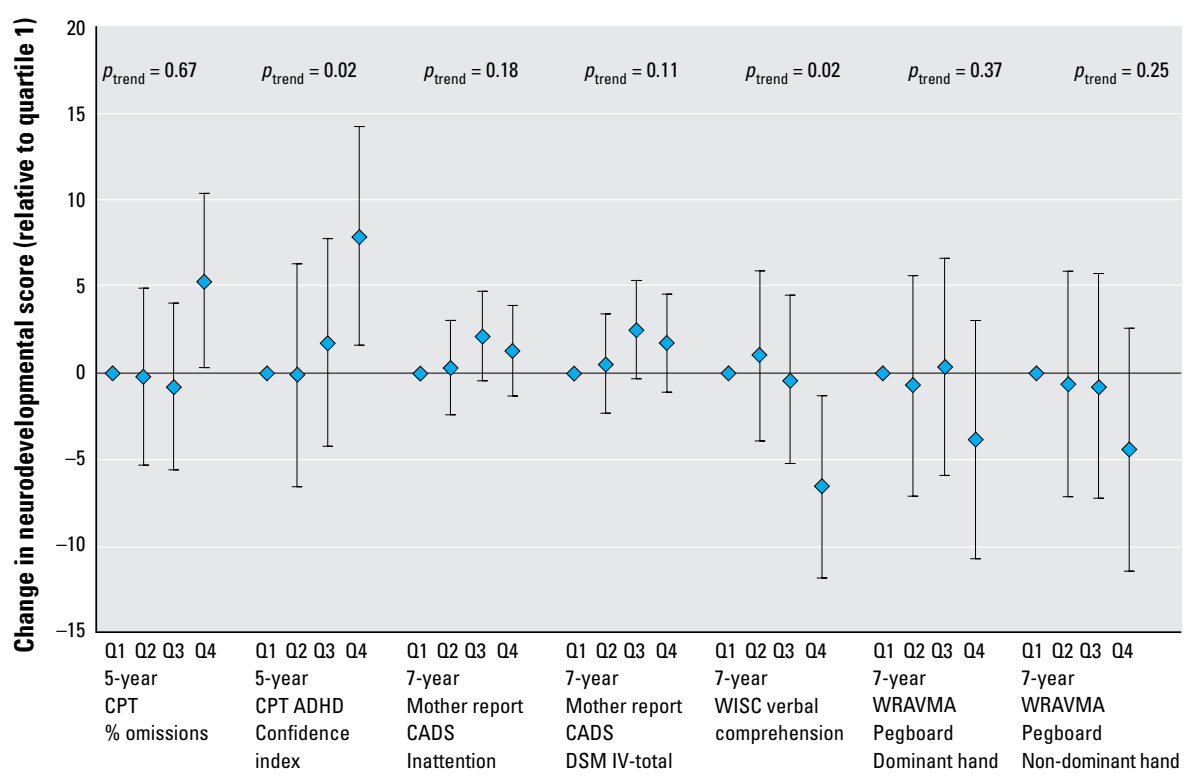

Figure 1. The point estimate and $95 \% \mathrm{Cl}$ for each quartile (0) of maternal $\sum$ PBDE concentration for outcomes that showed overall associations and evidence of nonlinearity (at $p<0.1$ ). The quartile ranges for maternal PBDEs were $\leq 14.4,14.5-24.78,24.8-41.97$, and $\geq 42 \mathrm{ng} / \mathrm{g}$ lipid. Tests for trend come from models using PBDE quartile (1-4) as a continuous variable.

Child $\Sigma$ PBDEs were marginally related to nondominant hand pegboard performance at age 7 years, but not with other motor outcomes.

Cognitive functioning. We observed no associations between maternal $\Sigma$ PBDE concentrations and child PPVT/TVIP or WPPSI Performance IQ scores at age 5 years (Table 3). However, at age 7 years, maternal $\Sigma$ PBDEs were associated with significant decrements in WISC Verbal Comprehension IQ, contributing to a somewhat lowered FullScale IQ. Quartile analysis indicated that the association was primarily driven by a Verbal Comprehension IQ decrement in the highest quartile $(\beta=-6.0 ; 95 \% \mathrm{CI}:-11.3,-0.7$; see Figure 1).

Children's $\Sigma$ PBDE concentrations were also related to Full-Scale IQ at age 7 years $(\beta=-5.6$; 95\% CI: $-10.8,-0.3)$, particularly with the Perceptual Reasoning IQ, Processing Speed IQ, and Verbal Comprehension IQ subscales (Table 3 ).

Sensitivity analyses. The above relationships were not confounded by maternal lead, PCB, or OP pesticide exposures, or substantially altered when controlled (in separate models) for birth weight, gestational age, or prenatal thyroid hormones. Overall, associations with individual PBDE congeners or the sum of all 10 congeners [see Supplemental Maternal, Table S6 (http:// dx.doi.org/10.1289/ehp.1205597)] were generally consistent with results for the sum of the four major congeners. Depending on the outcome, there were between 0 and 4 outliers with respect to either $\Sigma$ PBDE concentrations or outcomes; excluding them did not substantively affect the results (data not shown). Except where noted, we did not find evidence of effect modification by child sex.

When both maternal and child $\Sigma$ PBDE levels were entered into the same model $(n=214)$, associations were attenuated (data not shown) but child $\Sigma$ PBDE levels were still associated with a borderline increase in teacher-reported scores for inattention on the BASC ( $\beta=2.8 ; 95 \% \mathrm{CI}:-0.2,5.7)$ and maternal $\Sigma$ PBDE levels were still associated with maternally-reported CADS DSM-IV Total scale scores $(\beta=2.6 ; 95 \% \mathrm{CI}:-0.3$, 5.5), decreased Verbal Comprehension IQ $(\beta=-5.2 ; 95 \% \mathrm{CI}:-10.4,0.1)$ and FullScale IQ ( $\beta=-5.2 ; 95 \%$ CI: $-10.6,0.1)$, and lower nondominant hand pegboard scores $(\beta=-6.5 ; 95 \% \mathrm{CI}:-13.4,0.3)$.

\section{Discussion}

In the present study, we report associations between mothers' prenatal serum concentrations of PBDEs and evidence of deficits in attention, fine motor coordination, and cognitive functioning (particularly verbal comprehension) in their children at ages 5 and/or 7 
years. Despite only weak correlations between PBDE concentrations in maternal prenatal and child age 7 years blood, we found associations between cognition, motor function and attention with both maternal and child PBDE exposures. The observed results appeared to be independent of associations previously reported in this cohort between maternal PBDEs and maternal thyroid hormone (Chevrier et al. 2010) or child birth weight (Harley et al. 2011) and between maternal organophosphate pesticide exposure and child neurobehavioral development (Bouchard et al. 2011; Eskenazi et al. 2007; Marks et al. 2010). In addition, associations were not confounded by maternal lead or PCB levels, which were at low background levels.

This is the largest study to date on the potential neurodevelopmental impacts of
PBDE exposures, and largely supports findings from three smaller studies, including those with substantially lower PBDE serum levels (Gascon et al. 2011; Herbstman et al. 2010; Hoffman et al. 2012; Roze et al. 2009). Our results are also similar to those reported between prenatal exposure to $\mathrm{PCB}$, which are chemically similar to PBDEs, and poorer attention and cognition or mental development in children (Grandjean et al. 2001; Jacobson and Jacobson 2003; KoopmanEsseboom et al. 1996; Rogan and Gladen 1991; Sagiv et al. 2012).

A notable finding of our study is that, in addition to in utero exposures, childhood PBDE concentrations were also associated with neurodevelopmental deficits. Although we hypothesized a priori that prenatal exposure would be more influential than postnatal

Table 2. Adjusted linear models for motor function in CHAMACOS children at 5 and 7 years of age, per 10 -fold increase in maternal prenatal and child PPBDE concentration (ng/g, lipid-adjusted).

\begin{tabular}{|c|c|c|c|c|}
\hline \multirow[b]{2}{*}{ Outcome } & \multicolumn{2}{|c|}{ Maternal $\sum$ PBDE ${ }^{a, b}$} & \multicolumn{2}{|c|}{ Child $\sum$ PBDE ${ }^{b, c}$} \\
\hline & $n$ & $\beta(95 \% \mathrm{Cl})$ & $n$ & $\beta(95 \% \mathrm{Cl})$ \\
\hline \multicolumn{5}{|c|}{ Assessment of 5-year-olds } \\
\hline \multicolumn{5}{|c|}{ WRAVMA pegboard (standard score) } \\
\hline Dominant hand & 254 & $-4.3(-9.6,1.0)$ & & \\
\hline Nondominant hand & 252 & $-5.6(-10.8,-0.4)^{* *, \# \#}$ & & \\
\hline \multicolumn{5}{|c|}{ Finger tap (BARS $z$-score) } \\
\hline Dominant hand & 234 & $-0.4(-0.7,0.0)^{* *}$ & & \\
\hline Nondominant hand & 234 & $-0.2(-0.5,0.1)$ & & \\
\hline \multicolumn{5}{|l|}{ McCarthy (z-score) } \\
\hline Gross motor leg & 241 & $0.0(-0.3,0.4)^{\#}$ & & \\
\hline Bean bag catch & 249 & $-0.1(-0.4,0.2)^{\#}$ & & \\
\hline \multicolumn{5}{|c|}{ Assessment of 7-year-olds } \\
\hline \multicolumn{5}{|c|}{ WRAVMA pegboard (standard score) } \\
\hline Dominant hand & 258 & $-5.4(-11.1,0.3)^{*, \#, \# \#}$ & 269 & $-5.4(-12.0,1.2)$ \\
\hline Nondominant hand & 258 & $-6.5(-12.3,-0.7)^{* * * \#}$ & 268 & $-6.1(-12.7,0.4)^{*}$ \\
\hline \multicolumn{5}{|c|}{ Finger tap (BARS z-score) } \\
\hline Dominant hand & 258 & $-0.1(-0.4,0.2)^{\#}$ & 269 & $-0.2(-0.6,0.2)$ \\
\hline Nondominant hand & 258 & $-0.1(-0.4,0.2)^{\#}$ & 268 & $-0.1(-0.5,0.2)$ \\
\hline \multicolumn{5}{|l|}{ McCarthy (z-score) } \\
\hline Gross motor leg & 255 & $-0.1(-0.4,0.1)^{\#}$ & 266 & $-0.1(-0.4,0.2)^{\#}$ \\
\hline Bean bag catch & 258 & $0.0(-0.3,0.4)$ & 268 & $0.0(-0.4,0.3)$ \\
\hline
\end{tabular}

${ }^{a}$ Maternal PBDE models control for child's age, sex, home score at 6-month visit, father living with family, handedness, location of testing, whether the child attended preschool, maternal years in United States before giving birth, and psy-

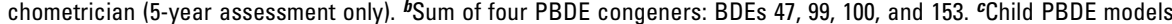
control for child's age, sex, home score at 7 -year visit, and location of testing. ${ }^{*} p<0.1 .{ }^{* *} p \leq 0.05$. ${ }^{*}$ Digression from linearity at $p<0.10{ }^{\# \#}$ Interaction with child sex at $p<0.10$.

Table 3. Adjusted linear models for measures of cognition at 5 and 7 years of age (standard score), per 10 -fold increase in maternal prenatal and child $\sum$ PBDE concentration (ng/g, lipid-adjusted).

\begin{tabular}{|c|c|c|c|c|}
\hline \multirow[b]{2}{*}{ Outcome } & \multicolumn{2}{|c|}{ Maternal $\sum \mathrm{PBDE}^{a, b}$} & \multicolumn{2}{|r|}{ Child $\sum \mathrm{PBDE}^{b, c}$} \\
\hline & $n$ & $\beta(95 \% \mathrm{Cl})$ & $n$ & $\beta(95 \% \mathrm{Cl})$ \\
\hline \multicolumn{5}{|l|}{ Assessment of 5-year-olds } \\
\hline PPVT & 252 & $0.4(-5.1,5.9)$ & & \\
\hline Performance 10 & 256 & $0.9(-3.5,5.3)$ & & \\
\hline \multicolumn{5}{|l|}{ Assessment of 7-year-olds } \\
\hline Full-Scale IO & 231 & $-4.7(-9.4,0.1)^{*}$ & 248 & $-5.6(-10.8,-0.3)^{* *}$ \\
\hline Verbal Comprehension I0 & 258 & $-5.5(-10.0,-1.0)^{* *, \#}$ & 269 & $-4.3(-9.4,0.8)^{*}$ \\
\hline Perceptual Reasoning IO & 258 & $-2.4(-7.6,2.9)$ & 269 & $-5.2(-11.1,0.7)^{*}$ \\
\hline Working Memory IO & 231 & $-2.4(-7.2,2.3)^{\#}$ & 249 & $-2.3(-7.4,2.8)$ \\
\hline Processing Speed IO & 232 & $-2.3(-6.8,2.3)$ & 249 & $-6.6(-11.4,-1.8)^{* *}$ \\
\hline
\end{tabular}

${ }^{a}$ Maternal PBDE models control for child's age, sex, home score at 6-month visit, language of assessment, and maternal years living in United States before giving birth. 'b ${ }^{5}$ um of four PBDE congeners: BDEs 47, 99, 100, and 153. ${ }^{c}$ Child PBDE models control for child's age, sex, home score at 7-year visit, maternal PPVT, language of examination, maternal years living in the United States before giving birth, parity, and prenatal exposure to environmental tobacco smoke. ${ }^{*} p<0.1$. ${ }^{* *} p \leq 0.05$. "Digression from linearity at $p<0.10$. exposure, the 7-year-olds' average PBDE concentrations were much higher than those in their mothers during pregnancy; we attribute this difference in part to the lifetime residence of the children in California compared with mothers, many of whom were recent immigrants to California when their levels were measured (Eskenazi et al. 2011).

In animal studies, PBDE exposure has been associated with increased death of cerebellar granule cells, alterations in neuronal arachidonic acid release, and disruption of calcium homeostasis (Birnbaum and Staskal 2004). Other potential mechanisms include perturbations of the cholinergic neurotransmitter system, interference with cellular signaling (Viberg et al. 2002a, 2002b, 2003), and, because of PBDEs' structural similarity to $\mathrm{T}_{4}$, effects on maternal thyroid hormone necessary for normal infant brain development (Darnerud et al. 2007; Richardson et al. 2008; Zhou et al. 2002). However, maternal thyroid hormone did not appear to explain the associations observed in our study population, as adding it to models did not measurably change the results.

Important strengths of the current study include its longitudinal design and use of comprehensive neurobehavioral assessments, which incorporate input from multiple informants. Limitations of this study are that we did not observe consistency in associations with PBDEs across informants for measures of attention (although their responses were moderately correlated), and we constructed numerous statistical models (although performance across domains was also moderately correlated), which increased the possibility of a chance finding. We also did not measure some higher-brominated compounds (e.g., BDE-209), which are present in decaBDE. Another study, however, indicates that BDE-209 represents a very small fraction of total serum PBDE concentrations in a different population of California children (Rose et al. 2010).

\section{Conclusions}

This study's finding of significant associations of both maternal prenatal and childhood PBDE exposures with poorer attention, fine motor coordination, and cognition in early school-age children contributes to the growing evidence of adverse associations between PBDE exposure and children's neurobehavioral development. Although these results are of particular concern for California children, they are also relevant to other locations, many of which contain products manufactured to meet California's standards. With the phaseout of pentaBDE, other flame retardants have been used to achieve compliance with TB 117 . Additional research is needed to determine the potential child health consequences of these new chemical flame retardants. 


\section{REFERENCES}

Achenbach T, Rescorla L. 2000. Manual for the ASEBA Preschool Forms \& Profiles. Burlington, VT:University of Vermont, Research Center for Children, Youth, and Families.

Adams W, Sheslow D. 1995. Wide Range Assessment of Visual Motor Abilities (WRAVMA). Wilmington, DE:Wide Range, Inc.

American Psychiatric Association. 1994. Diagnostic and Statistical Manual of Mental Disorders, 4th ed. Washington, DC:American Psychiatric Association.

Barr JR, Maggio VL, Barr DB, Turner WE, Sjödin A, Sandau CD, et al. 2003. New high-resolution mass spectrometric approach for the measurement of polychlorinated biphenyls and organochlorine pesticides in human serum. J Chromatogr B Analyt Technol Biomed Life Sci 794(1):137-148.

Birnbaum LS, Staskal DF. 2004. Brominated flame retardants: cause for concern? Environ Health Perspect 112:9-17.

Bouchard MF, Chevrier J, Harley KG, Kogut K, Vedar M, Calderon N, et al. 2011. Prenatal exposure to organophosphate pesticides and 10 in 7-year-old children. Environ Health Perspect 119:1189-1195.

Bradman A, Castorina R, Sjödin A, Fenster L, Jones RS, Harley KG, et al. 2012. Factors associated with serum polybrominated diphenyl ether (PBDE) levels among school-age children in the CHAMACOS cohort. Environ Sci Technol 46(13):7373-7381.

Bradman A, Eskenazi B, Barr D, Bravo R, Castorina R, Chevrier J, et al. 2005. Organophosphate urinary metabolite levels during pregnancy and after delivery in women living in an agricultural community. Environ Health Perspect 113:1802-1807.

Bravo R, Driskell WJ, Whitehead RD Jr, Needham LL, Barr DB. 2002. Quantitation of dialkyl phosphate metabolites of organophosphate pesticides in human urine using GC-MS-MS with isotopic internal standards. J Anal Toxicol 26(5):245-252

Caldwell B, Bradley R. 1984. Home Observation for Measurement of The Environment. Little Rock, AR:University of Arkansas.

California Department of Consumer Affairs. 2000. Technical Bulletin 117: Requirements, Test Procedure and Apparatus for Testing the Flame Retardance of Resilient Filling Materials Used in Upholstered Furniture. Available: http:// www.bhfti.ca.gov/industry/117.pdf [accessed 23 June 2010].

Castorina R, Bradman A, Sjödin A, Fenster L, Jones RS, Harley KG, et al. 2011. Determinants of serum polybrominated diphenyl ether (PBDE) levels among pregnant women in the CHAMACOS cohort. Environ Sci Technol 45(15):6553-6560.

Chao HR, Wang SL, Lee WJ, Wang YF, Papke 0. 2007. Levels of polybrominated diphenyl ethers (PBDEs) in breast milk from central Taiwan and their relation to infant birth outcome and maternal menstruation effects. Environ Int 33(2):239-245.

Chevrier J, Harley KG, Bradman A, Gharbi M, Sjödin A, Eskenazi B. 2010. Polybrominated diphenyl ether (PBDE) flame retardants and thyroid hormone during pregnancy. Environ Health Perspect 118:1444-1449.

Conners CK. 2001. Conner's Rating Scales-Revised (CSR-R) Technical Manual (includes auxilliary scales: CADS-P and CADS-T). North Tonawanda, NY:Multi-Health Systems Inc.

Conners CK, Staff M. 2001. Conners' Kiddie Continuous Performance Test (K-CPT): Computer Program for Windows Technical Guide and Software Manual. Toronto, ON, Canada:Multi-Health Systems Inc.

Darnerud PO, Aune M, Larsson L, Hallgren S. 2007. Plasma PBDE and thyroxine levels in rats exposed to Bromkal or BDE-47. Chemosphere 67(9):S386-S392.

Dunn L, Dunn L. 1981. Peabody Picture Vocabulary Test, Revised. Circle Pines, MN:American Guidance Service.

Eskenazi B, Fenster L, Castorina R, Marks AR, Sjödin A, Rosas LG, et al. 2011. A comparison of PBDE serum concentrations in Mexican and Mexican-American children living in California. Environ Health Perspect 119:1442-1448.

Eskenazi B, Harley K, Bradman A, Weltzien E, Jewell NP, Barr DB, et al. 2004. Association of in utero organophosphate pesticide exposure and fetal growth and length of gestation in an agricultural population. Environ Health Perspect 112:1116-1124.

Eskenazi B, Marks AR, Bradman A, Fenster L, Johnson C, Barr DB, et al. 2006. In utero exposure to dichlorodiphenyltrichloroethane (DDT) and dichlorodiphenyldichloroethylene (DDE) and neurodevelopment among young Mexican American children. Pediatrics 118(1):233-241.

Eskenazi B, Marks AR, Bradman A, Harley K, Barr DB Johnson C, et al. 2007. Organophosphate pesticide exposure and neurodevelopment in young Mexican-American children. Environ Health Perspect 115:792-798.

Gascon M, Fort M, Martinez D, Carsin AE, Forns J, Grimalt JO et al. 2012. Polybrominated diphenyl ethers (PBDEs) in breast milk and neuropsychological development in infants. Environ Health Perspect 120:1760-1765.

Gascon M, Vrijheid M, Martinez D, Forns J, Grimalt J0, Torrent $\mathrm{M}$, et al. 2011. Effects of pre and postnatal exposure to low levels of polybromodiphenyl ethers on neurodevelopment and thyroid hormone levels at 4 years of age. Environ Int 37(3):605-611.

Geyer HJ, Schramm K-W, Darnerud PO, Aune M, Feicht A Fried KW. 2004. Terminal elimination half-lives of the brominated flame retardants TBBPA, HBCD, and lower brominated PBDEs in humans. Organohalogen Compounds 66:3867-3872

Grandjean P, Weihe P, Burse VW, Needham LL, StorrHansen $E$, Heinzow B, et al. 2001. Neurobehavioral deficits associated with PCB in 7-year-old children prenatally exposed to seafood neurotoxicants. Neurotoxicol Teratol 23(4):305-317.

Harley KG, Chevrier J, Schall RA, Sjödin A, Bradman A, Eskenazi B. 2011. Association of prenatal exposure to polybrominated diphenyl ethers and infant birth weight. Am J Epidemiol 174(8):885-892.

Herbstman JB, Sjödin A, Apelberg BJ, Witter FR, Halden RU, Patterson DG, et al. 2008. Birth delivery mode modifies the associations between prenatal polychlorinated bipheny PCB) and polybrominated diphenyl ether (PBDE) and neonatal thyroid hormone levels. Environ Health Perspect 116:1376-1382.

Herbstman JB, Sjödin A, Kurzon M, Lederman SA, Jones RS, Rauh V, et al. 2010. Prenatal exposure to PBDEs and neurodevelopment. Environ Health Perspect 118:712-719.

Hoffman K, Adgent M, Goldman BD, Sjödin A, Daniels JL. 2012. Lactational exposure to polybrominated diphenyl ethers and its relation to social and emotional development among toddlers. Environ Health Perspect 120:1438-1442.

Jacobson JL, Jacobson SW. 2003. Prenatal exposure to polychlorinated biphenyls and attention at school age. J Pediatr 143(6):780-788.

Koopman-Esseboom C, Weisglas-Kuperus N, de Ridder MA, Van der Paauw CG, Tuinstra LG, Sauer PJ. 1996. Effects of polychlorinated biphenyl/dioxin exposure and feeding type on infants' mental and psychomotor development. Pediatrics 97(5):700-706

Lubin JH, Colt JS, Camann D, Davis S, Cerhan JR, Severson RK, et al. 2004. Epidemiologic evaluation of measurement data in the presence of detection limits. Environ Health Perspect 112:1691-1696.

Marks AR, Harley K, Bradman A, Kogut K, Barr DB, Johnson C, et al. 2010. Organophosphate pesticide exposure and attention in young Mexican-American children: the CHAMACOS study. Environ Health Perspect 118:1768-1774.

McCarthy D. 1972. McCarthy Scales of Children's Abilities. New York:Psychological Corporation.

Nelson JC, Tomei RT. 1988. Direct determination of free thyroxin in undiluted serum by equilibrium dialysis/radioimmunoassay. Clin Chem 34(9):1737-1744.

Noyes PD, Hinton DE, Stapleton HM. 2011. Accumulation and debromination of decabromodiphenyl ether (BDE-209) in juvenile fathead minnows (Pimephales promelas) induces thyroid disruption and liver alterations. Toxicol Sci 122(2):265-274.

Phillips DL, Pirkle JL, Burse VW, Bernert JT Jr, Henderson LO Needham LL. 1989. Chlorinated hydrocarbon levels in human serum: effects of fasting and feeding. Arch Environ Contam Toxicol 18(4):495-500.

Radloff L. 1977. The CES-D Scale: a self-report depression scale for research in the general population. Appl Psychol Meas 1(3):385-401.

Reynolds CR, Kamphaus RW. 2004. BASC-2: Behaviour Assessment System for Children, Second Edition Manual. Circle Pines, MN:AGS Publishing.

Richardson VM, Staskal DF, Ross DG, Diliberto JJ, DeVito MJ Birnbaum LS. 2008. Possible mechanisms of thyroid hormone disruption in mice by BDE 47 , a major polybrominated diphenyl ether congener. Toxicol Appl Pharmaco 226(3):244-250.

Rogan W, Gladen B. 1991. PCBs, DDE, and child development at 18 and 24 months. Ann Epidemiol 1(5):407-413.

Rohlman DS, Gimenes LS, Eckerman DA, Kang SK, Farahat FM, Anger WK. 2003. Development of the Behavioral Assessment and Research System (BARS) to detect and characterize neurotoxicity in humans. Neurotoxicology 24(4-5):523-531.

Rose M, Bennett DH, Bergman A, Fangstrom B, Pessah IN Hertz-Picciotto I. 2010. PBDEs in 2-5 year-old children from California and associations with diet and indoor environment. Environ Sci Technol 44(7):2648-2653.

Roze E, Meijer L, Bakker A, Van Braeckel KN, Sauer PJ, Bos AF. 2009. Prenatal exposure to organohalogens, including brominated flame retardants, influences motor, cognitive, and behavioral performance at school age. Environ Health Perspect 117:1953-1958.

Sagiv SK, Thurston SW, Bellinger DC, Altshul LM, Korrick SA 2012. Neuropsychological measures of attention and impulse control among 8-year-old children exposed prenatally to organochlorines. Environ Health Perspect 120:904-909.

Sjödin A, Jones RS, Lapeza CR, Focant JF, McGahee EE III, Patterson DG Jr. 2004. Semiautomated high-throughput extraction and cleanup method for the measurement of polybrominated diphenyl ethers, polybrominated biphenyls, and polychlorinated biphenyls in human serum. Anal Chem 76(7):1921-1927.

Sjödin A, Papke 0, McGahee E, Focant JF, Jones RS, Pless Mulloli T, et al. 2008. Concentration of polybrominated diphenyl ethers (PBDEs) in household dust from various countries. Chemosphere 73(1 suppl):S131-S136.

Stapleton HM, Kelly SM, Allen JG, McClean MD, Webster TF. 2008. Measurement of polybrominated diphenyl ethers on hand wipes: estimating exposure from hand-to-mouth contact. Environ Sci Technol 42(9):3329-3334.

Viberg $H$, Fredriksson A, Eriksson P. 2002a. Neonatal exposure to the brominated flame retardant 2,2',4,4',5-pentabromodiphenyl ether causes altered susceptibility in the cholinergic transmitter system in the adult mouse. Toxicol Sci 67(1):104-107.

Viberg $\mathrm{H}$, Fredriksson A, Eriksson P. 2002b. Neonatal exposure to a brominated flame retardant, $2,2^{\prime}, 4,4^{\prime}, 5,5^{\prime}$-hexabromodiphenyl ether (PBDE 153) affects behaviour and cholinergic nicotinic receptors in brain of adult mice [Abstract 643]. In: The Toxicologist: Proceedings of the 41st Annual Meeting of the Society of Toxicology, 17-21 March 2002, Nashville, TN. Reston, VA:Society of Toxicology, 132.

Viberg H, Fredriksson A, Eriksson P. 2003. Neonatal exposure to polybrominated diphenyl ether (PBDE 153) disrupts spontaneous behaviour, impairs learning and memory, and decreases hippocampal cholinergic receptors in adult mice. Toxicol Appl Pharmacol 192(2):95-106.

Wechsler D. 2003. Wechsler Intelligence Scale for Children-Fourth Edition (WISC-IV) Administration and Scoring Manual. San Antonio, TX:Harcourt Assessment Incorporated.

Zhou T, Taylor MM, DeVito MJ, Crofton KM. 2002. Developmental exposure to brominated diphenyl ethers results in thyroid hormone disruption. Toxicol Sci 66(1):105-116.

Zota AR, Rudel RA, Morello-Frosch RA, Brody JG. 2008. Elevated house dust and serum concentrations of PBDEs in California: unintended consequences of furniture flammability standards? Environ Sci Technol 42(21):8158-8164. 